\title{
Minimum Norm Solutions for Cooperative Games
}

\author{
Klaus Kultti \\ University of Helsinki and HECER \\ and \\ Hannu Salonen \\ University of Turku
}

Discussion Paper No. 67

May 2005

ISSN 1795-0562

HECER - Helsinki Center of Economic Research, P.O. Box 17 (Arkadiankatu 7), FI-00014 University of Helsinki, FINLAND, Tel +358-9-191-28780, Fax +358-9-191-28781, E-mail info-hecer@helsinki.fi, Internet www.hecer.fi 


\title{
Minimum Norm Solutions for Cooperative Games* $^{*}$
}

\begin{abstract}
We show that to each linear solution that has the inessential game property, there is an inner product on the space of games such that the solution to each game is the best additive approximation of the game (w.r.t. the norm derived from this inner product). If the space of games has an inner product, then the function that to each game assigns the best additive approximation of this game (w.r.t. to the norm derived from this inner product) is a linear solution that has the inessential game property. Both claims remain valid also if solutions are required to be efficient.
\end{abstract}

JEL Classification: C78

Keywords: Least squares, Banzhaf-index, additive approximation.

Klaus Kultti

Department of Economics

P.O. Box 17 (Arkadiankatu 7)

FI-00014 University of Helsinki

FINLAND

e-mail: klaus.kultti@helsinki.fi
Hannu Salonen

Department of Economics

University of Turku

FI-20014 Turku

FINLAND

e-mail: hansal@utu.fi

* We thank Hannu Nurmi for useful comments and the Yrjö Jahnsson Foundation for financial support. 


\section{Introduction}

The games we analyze are the familiar $\mathrm{n}$-person cooperative games with side payments. A solution is a function $f$ that to each game $v$ assigns an additive game $f(v)$. Additive games are like (signed) measures: each player is given a payoff, and the payoff to a coalition is just the sum of the payoffs of the players in that coalition. Additive games are also called "inessential games". A solution $f$ has the inessential game property, if the solution to any additive game $p$ is that game itself: $f(p)=p$. A solution $f$ is linear, if it is additive $(f(v+w)=f(v)+f(w)$, and satisfies $f(a v)=a f(v)$ for all real numbers $a$.

We show that to each linear solution that has the inessential game property, there is an inner product such that the solution to each game is the best additive approximation of the game (w.r.t. the norm derived from this inner product). Conversely, if the space of games has an inner product, then the function that to each game assigns the best additive approximation of this game (w.r.t. to the norm derived from this inner product) is a linear solution that has the inessential game property. Both claims remain valid also if solutions are required to be efficient.

Some of the best known solutions for cooperative games, such as the Shapley value and the Banzhaf value, satisfy linearity and have the inessential game property. Classes of solutions such as semivalues (Dubey, Neyman and Weber (1981) and least square values (Ruiz, Valenciano and Zarzuelo (1996, 1998)) satisfy these conditions as well. So all these solutions can be viewed as "minimum norm solutions". Given a norm on the space of games, the minimum norm solution to a game is the solution that is closest to the game w.r.t. the norm. In this paper we consider only such norms that can be derived from some inner product.

Minimum norm solutions have some intuitive appeal. A game $v$ specifies the worth of each coalition. Potential solutions to a game are additive games. Find the additive game $p$ that is as close as possible to the given game $v$. Then the payoffs $p_{i}$ to players $i$ should reflect the importance of these players in $v$ as well as possible. If the norm is changed, then another solution $p^{\prime}$ to $v$ is found. Something like this may happen in real world as well: if social norms change, then the characteristics of individuals that earlier were deemed valuable may become less valuable.

The additivity property of solutions has received some criticism in the literature. But this property is unavoidable if we seek for minimum norm solutions since linear solutions are additive. Therefore, if we don't accept additivity, we cannot find the idea behind the minimum norm solutions very interesting either. Conversely, if linearity is accepted as a reasonable axiom, then minimum norm solutions should be accepted as well. (Unless, of course, 
one feels strongly that the inessential game property is counterintuitive.)

The paper is organized in the following way. In Section 2 notation is introduced. In Section 3 we mainly review some results about least square solutions. The main results are given in Section 4. Section 4 contains some results concerning the core.

\section{Preliminaries}

Given a nonempty finite player set $N=\{1, \ldots, n\}$, an $n$-person cooperative game is a function $v$ that assigns to each coalition $S \subset N$ a real number $v(S)$ with the convention $v(\emptyset)=0$. Hence a game $v$ may be viewed as a vector in the linear space $\mathbb{R}^{2^{N}-1}$. We denote by $v_{i}$ the coordinate of $v$ corresponding to the singleton coalition $\{i\}$. We denote by $\mathbb{V}$ the space $\mathbb{R}^{2^{N}-1}$ of all $n$-person games. The origin of $\mathbb{V}$ is denoted by $\overline{0}$.

An inner product on $\mathbb{V}$ is a function $\langle\cdot, \cdot\rangle: \mathbb{V} \times \mathbb{V} \longrightarrow \mathbb{R}$ such that for all games $v, v^{\prime}, w:(1)\langle v, w\rangle=\langle w, v\rangle ;(2)\left\langle a v+b v^{\prime}, w\right\rangle=a\langle v, w\rangle+b\left\langle v^{\prime}, w\right\rangle$, for all $a, b \in \mathbb{R} ;(3)\langle v, v\rangle \geq 0 ;(4)\langle v, v\rangle=0$ implies $v=\overline{0}$. If the condition (4) is not satisfied, then $\langle\cdot, \cdot\rangle$ is a semi inner product. A norm (seminorm) $\|\cdot\|$ on $\mathbb{V}$ can be defined from an inner product (semi inner product) $\langle\cdot, \cdot\rangle$ by $\|v\|=$ $\sqrt{\langle v, v\rangle}$. A metric (semimetric) $d$ can be defined from a norm (seminorm) by $d(v, w)=\|v-w\|$. Interpreting $v$ as a column vector, its transpose $v^{T}$ is a row vector. Then the usual dot product $v^{T} w=\sum_{S \subset N} v(S) w(S)$ is an inner product. The norm derived from this inner product is the Euclidean norm, and the metric derived from this norm is the Euclidean metric.

A game $v \in V$ is additive, if $v(S)=\sum_{i \in S} v_{i}$, for all $S \subset N$. We denote by $\mathbb{A}$ the set of all additive $n$-person games, and note that it is a linear subspace of $\mathbb{V}$. A game $v \in \mathbb{V}$ is superadditive, if $v(S \cup T) \geq v(S)+v(T)$, when $S \cap T=\emptyset$. Additive games are superadditive. A game $v \in \mathbb{V}$ is convex, if $v(S)-v(S \backslash\{i\}) \geq v(T)-v(T \backslash\{i\})$, for all $i \in N$ and for all coalitions $S, T$ such that $i \in T \subset S$. Convex games are superadditive. A game $v \in \mathbb{V}$ is monotone, if $v(S) \leq v(T)$, when $S \subset T$. Monotone games are positive $(v(S) \geq 0$, for all $S)$, and positive superadditive games are monotone.

A solution to $n$-person cooperative games is a function $f: \mathbb{V} \longrightarrow \mathbb{A}$ that to each game $v$ associates an additive game $f(v)$. The best-known solution is the Shapley value $\phi$ :

$$
\phi(v)_{i}=\sum_{S \ni i} \frac{(n-s) !(s-1) !}{n !}[v(S)-v(S \backslash\{i\})],
$$


for all $i \in N$, where $s=|S|$ denotes the cardinality of coalition $S$. Let $\pi: N \longrightarrow N$ be any bijection. To each $v \in \mathbb{V}$ define a game $\pi v$ by $\pi v(\pi(S))=v(S)$, for all $S \subset N$, where $\pi(S)=\{\pi(i) \mid i \in S\}$. The Shapley value is the only solution $f$ on $\mathbb{V}$ satisfying the following four conditions (Shapley 1953; Winter 2002). The conditions must hold for every game $v \in \mathbb{V}$, but we don't repeat this in the statements of the conditions.

A1. (Efficiency) $f(v)(N)=v(N)$.

A2. (Anonymity) $f(\pi v)_{\pi(i)}=f(v)_{i}$, for all $i \in N$, and any bijection $\pi: N \longrightarrow N$.

A3. (Dummy) If $v(S)-v(S \backslash\{i\})=0$ for all $S \subset N$, then $f(v)_{i}=0$.

A4. (Additivity) $f(v+w)=f(v)+f(w)$.

Another well-known solution is the Banzhaf value $\beta$ :

$$
\beta(v)_{i}=\frac{1}{2^{n-1}} \sum_{S \ni i}[v(S)-v(S \backslash\{i\})],
$$

for all $i \in N$. It satisfies all the properties mentioned above except efficiency. Both the Shapley value and the Banzhaf value satisfy the following linearity condition, which is a bit stronger than additivity.

A5. (Linearity) $f(a v+b w)=a f(v)+b f(w)$, for all $a, b \in \mathbb{R}$.

Both the Shapley value and the Banzhaf value satisfy the following condition.

A6. (Inessential game) $f(v)=v$, for all additive games $v$.

We need also the following axioms in the sequel.

A7. (Coalitional mononicity) If $v(S)>w(S)$ and $v(T)=w(T)$ for all $T \neq S$, then $f(v)_{i} \geq f(w)_{i}$ for all $i \in S$.

A8. (Positivity) If $v$ is monotonic, then $f(v) \geq \overline{0}$.

The core $C(v)$ of a game $v$ consists of additive games $x \in \mathbb{A}$ such that $x(N)=v(N)$ and $x(S) \geq v(S)$ for all $S \subset N$. In general the core may be 
empty, but the Shapley value is in the core of any convex game.

\section{Least square values}

Ruiz, Valenciano and Zarzuelo $(1996,1998)$ study least square values, which they define in the following way. Let $m \in \mathbb{V}$ be a nonnegative game (vector) such that $m(S)>0$ for some $S$, and $m(S)=m(T)$ if $|S|=|T|$. Given a game $v \in \mathbb{V}$ and weights $m$, the least square value $L^{m}$ solves the following minimization problem;

$$
\operatorname{Min} \sum_{S \subset N} m(S)(v(S)-x(S))^{2} \text {, s.t. } x \in \mathbb{A}, x(N)=v(N)
$$

A solution is a least square value, if and only if it satisfies efficiency, linearity, symmetry, inessential game, and coalitional monotonicity (Ruiz, Valenciano and Zarzuelo 1998, Theorem 8, p.116). (Symmetry is a slightly weaker condition than anonymity: the result is true also if symmetry is replaced by anonymity.)

A semivalue (Dubey, Neyman and Weber 1981) is a solution that satisfies linearity, anonymity, inessential game and positivity. If $f$ is a semivalue, then it gives player $i \in N$ the payoff

$$
f(v)_{i}=\sum_{S \not \supset i} p(S)[v(S \cup\{i\})-v(S)],
$$

where $p$ is a probability measure over coalitions $S \neq N$ such that $|S|=|T|$ implies $p(S)=p(T)$.

Ruiz, Valenciano and Zarzuelo (1998, p. 120) observe that the additive normalization of a semivalue is in fact a least square value. That is, if $f$ is a semivalue, then $\tilde{f}(v)=f(v)+[v(N)-f(v)(N)] / n$ is the additive normalization of $f$, and $\tilde{f}$ is a least square value. They also remark that there are least square values $L^{m}$ that cannot be derived from any semivalue in this way.

If the weights for all coalitions are the same, then the solution to formula (1) minimizes the Euclidean distance between $v$ and the set of all additive games $x$ such that $v(N)=\sum_{i \in N} x_{i}$. So we can interpret the least square value as being the best additive approximation of a given game $v$. 
Similar interpretation applies also when the weights for coalitions of different sizes are not equal. In this case the distance between games is not given by the Euclidean metric but by some other metric. To see this, let $M$ be the diagonal matrix whose diagonal element corresponding to the coalition $S$ is $M_{S, S}=m(S)$. Interpret games $x \in \mathbb{V}$ as column vectors and their transposes $x^{T}$ as row vectors. Then $\langle x, y\rangle=x^{T} M y$ defines a semi inner product on $\mathbb{V}$ from which a seminorm $\|\cdot\|$ can be derived as explained in the previous section. Then $L^{m}(v)$ minimizes $\|v-p\|$ among all additive $p$ such that $p(N)=v(N)$.

Ruiz, Valenciano and Zarzuelo offer a different interpretation for the least square values. Their interpretation is related to another solution concept, the nucleolus (Schmeidler, 1969). Given a game $v$, the excess for coalition $S$ at $x \in \mathbb{A}$ is given by $e(S, x)=v(S)-x(S)$. Let $e(x)$ be the average of the excesses of all coalitions, and minimize $\sum_{S \subset N} m(S)(e(S, x)-e(x))^{2}$, s.t. $x \in$ $\mathbb{A}, x(N)=v(N)$. Ruiz, Valenciano and Zarzuelo note that in fact the solution to this problem is the same as the solution to the problem given in formula (1), so they minimize the (weighted) sum of squared excesses. The nucleolus, on the other hand, is found by minimizing lexicographically the maximal excess among all coalitions, and the solution is searched from the set of all imputations: $x \in \mathbb{A}, x(N)=v(N), x_{i} \geq v_{i}$, for all $i \in N$.

\subsection{Banzhaf, Shapley and least square values}

Let us seek the least square solution for games, when the weights for all coalitions are the same in formula (1), and denote this solution by $L$. So we may set these weights equal to unity, and our problem is to find the solution $L(v) \in \mathbb{A}$ that minimizes the inner product $(v-p)^{T}(v-p)$ subject to $p \in \mathbb{A}, p(N)=v(N)$.

Let us denote by $\mathbb{A}^{\perp}$ the orthogonal complement of $\mathbb{A}$ with respect to the dot product. That is, $\mathbb{A}^{\perp}=\left\{v \in \mathbb{V} \mid v^{T} p=0\right.$, for all $\left.p \in \mathbb{A}\right\}$. Any game $v \in \mathbb{V}$ can be uniquely expressed as the sum $v=v^{\perp}+p^{v}$, where $v^{\perp} \in \mathbb{A}^{\perp}$ and $p^{v} \in \mathbb{A}$.

Since $p^{v}$ is by definition the additive game that is closest to $v$, the Pythagorean Theorem says that $L(v)$ is the orthogonal projection of $p^{v}$ on the linear manifold $M=\{p \in \mathbb{A} \mid p(N)=v(N)\}$. (Note that the additivity of $L$ follows immediately from this observation, since projections into subspaces are linear mappings.)

So $L(v)=p$, where $p$ minimizes the dot product $\left(p-p^{v}\right)^{T}\left(p-p^{v}\right)$. In other words, the additive game $L(v)-p^{v}$ is closest to the origin among all additive games in the linear manifold $M^{\prime}=\left\{p \in \mathbb{A} \mid p(N)=v(N)-p^{v}(N)\right\}$. 
By symmetry of the Euclidean norm, $L(v)-p^{v}=\left(v(N)-p^{v}(N)\right) u$, where $u$ is the uniform additive game: $u(S)=s / n$ for all $S \subset N$.

In words, $L(v)_{i}$ is obtained by adding the amount $\left[v(N)-p^{v}(N)\right] / n$ to $p_{i}^{v}$. Since $L$ is additive, and $v=v^{\perp}+p^{v}$, we have $L\left(v^{\perp}\right)=\left(v(N)-p^{v}(N)\right) u$. So the value $L\left(v^{\perp}\right)$ is obtained by dividing the sum $v^{\perp}(N)$ evenly between players.

The Banzhaf value $\beta$ is additive, and satisfies $\beta(p)=p$ for all additive games $p$. Hence $\beta(v)=\beta\left(v^{\perp}\right)+p^{v}$. Let us calculate $\beta\left(v^{\perp}\right)$. Define for each $i \in N$ the additive game $\delta^{i}$ by $\delta^{i}(S)=1$ if $i \in S$, and $\delta^{i}(S)=0$ if $i \notin S$. Since $\left(v^{\perp}\right)^{T} \delta^{i}=0$ and $\left(v^{\perp}\right)^{T} \delta^{i}=\sum_{S \ni i} v^{\perp}(S)$ for all $i$, we have that $\sum_{S \subset N} v^{\perp}(S)=\sum_{S \ni i}\left[v^{\perp}(S)+v^{\perp}(S \backslash\{i\})\right]=\sum_{S \ni i}\left[v^{\perp}(S \backslash\{i\})\right]$. Hence $-\sum_{S \ni i} v^{\perp}(S \backslash\{i\})=-\sum_{S \subset N} v^{\perp}(S)$. So the Banzhaf value satisfies $\beta\left(v^{\perp}\right)_{i}=$ $-2^{1-n} \sum_{S \subset N} v^{\perp}(S)$, i.e.

$$
\beta\left(v^{\perp}\right)=\frac{-n}{2^{n-1}}\left[\sum_{S \subset N} v^{\perp}(S)\right] u
$$

That is, the Banzhaf value of $v^{\perp}$ divides the sum $\frac{-n}{2^{n-1}}\left[\sum_{S \subset N} v^{\perp}(S)\right]$ evenly between players. Define the additive normalization $\tilde{\beta}$ of the Banzhaf value by $\tilde{\beta}(v)_{i}=\beta(v)_{i}+[v(N)-\beta(v)(N)] / n$, for all games $v$ and all players $i \in N$. Then $\beta$ is clearly additive, and satisfies $\tilde{\beta}(p)=p$, for additive games $p$. So $\tilde{\beta}(v)=\tilde{\beta}\left(v^{\perp}\right)+p^{v}$. But $\tilde{\beta}\left(v^{\perp}\right)$ divides the sum $v^{\perp}(N)$ evenly between players, as does the least square value $L\left(v^{\perp}\right)$. We have

Theorem $1 L=\tilde{\beta}$.

Theorem 1 follows from the more general Theorem 12 by Ruiz, Valenciano and Zarzuelo (1998), but we gave a separate (and different) proof for completeness, and to help the reader to understand our results in the next section. Let us solve $p^{v}$, the orthogonal projection of $v$ onto $\mathbb{A}$.

Since $v^{\perp}=v-p^{v}$ and $\left(v^{\perp}\right)^{T} \delta^{i}=0$ we have $\left(v-p^{v}\right)^{T} \delta^{i}=0$ for all $i \in N$. That is,

$$
\sum_{S \ni i} v(S)=\sum_{S \ni i} p^{v}(S), \text { for all } i \in N
$$


But $i$ belongs to $2^{n-1}$ coalitions, and each $j \neq i$ belongs to exactly half of these coalitions. Hence

$$
\sum_{S \ni i} p^{v}(S)=2^{n-1} p_{i}^{v}+2^{n-2} p^{v}(N \backslash\{i\})
$$

because $\sum_{S \subset N} p(S)=2^{n-1} p(N)$ for all additive games $p$. Equations (3) and (4) imply

$$
p_{i}^{v}=\frac{1}{2^{n-2}} \sum_{S \ni i} v(S)-p^{v}(N), \text { for all } i \in N
$$

Summing up the equations in (5), we get

$$
p^{v}(N)=\frac{1}{(n+1) 2^{n-2}} \sum_{i=1}^{n} \sum_{S \ni i} v(S)
$$

Therefore by equations (4) and (5), we have

$$
p_{i}^{v}=\frac{1}{(n+1) 2^{n-2}}\left[(n+1) \sum_{S \ni i} v(S)-\sum_{j=1}^{n} \sum_{S \ni j} v(S)\right]
$$

The additive normalization $\tilde{\beta}$ of the Banzhaf value is obtained by adding the same constant to each players' $p_{i}^{v}$ given by (7). This is also the least square value by Theorem 1 . The ordinary Banzhaf value $\beta$ is obtained by adding another constant (but the same for all players) to the values $p_{i}^{v}$ given by (7). Note that $p_{i}^{v}<p_{j}^{v}$ for $i \neq j$ if and only if $\sum_{S \ni i} v(S)<\sum_{S \ni j} v(S)$. This has the following implications to the theory of voting games and power indices.

Recall that a game $v$ is simple, if $v(S)=0$ or $v(S)=1$, for all $S \subset N$. The coalitions $S$ such that $v(S)=1$ are called winning coalitions, and the remaining coalitions are called losing. If $v$ is simple, then $\sum_{S \ni i} v(S)$ is the number of those winning coalitions that include $i$. Solutions defined on simple games are usually called power indices. Best known power indices are the Shapley-Shubik index, and the Banzhaf power index. These are just the Shapley value and the Banzhaf value restricted to simple games. The least square values $L^{m}$ and the additive normalization $\tilde{\beta}$ of the Banzhaf value would also qualify as power indices, when restricted to simple games.

Theorem 2 Let $P$ be any power index such that for all simple games $v$ and all players $i, j \in N, P(v)_{i}<P(v)_{j}$ if and only if the number of winning 
coalitions containing $j$ is strictly greater than the number of winning coalitions containing $i$. Then for all simple games $v$ and all players $i, j \in N$, $P(v)_{i}<P(v)_{j}$ if and only if $\beta(v)_{i}<\beta(v)_{j}$.

From formula of the Banzhaf value, $\beta(v)_{i}=\frac{1}{2^{n-1}} \sum_{S \ni i}[v(S)-v(S \backslash\{i\})]$, it is clear that $\beta(v)_{i}<\beta(v)_{j}$, if $j$ has more swings. That is, there are more coalitions $S$ for $j$ such that $S$ is winning but $S \backslash\{j\}$ is loosing, than there are such coalitions for $i$. The following result is a direct consequence of Theorem 2 .

Corollary $1 \sum_{S \ni i} v(S)<\sum_{S \ni j} v(S)$ if and only if $j$ has more swings than $i$ in the simple game $v$.

Keane (1969) solved the minimization problem (1), and calculated the weights $m(S)$ for coalitions $S$ such that the solution is the Shapley value. These weights are given the following:

$$
m(S)=\frac{(n-s-1) !(s-1) !}{(n-1) !}, S \neq N .
$$

The weight $m(N)$ of the grand coalition turns out to be irrelevant in the minimization problem (1). Note that in three person games, these weights are the same for all coalitions, and therefore the Shapley value and the additive normalization of the Banzhaf value are the same on these games. Hence by Theorem $1, L=\phi$ on three person games.

\section{Main results}

Given an inner product $\langle\cdot, \cdot\rangle$ on $\mathbb{V}$, consider the following problem for each $v \in \mathbb{V}$.

$$
\operatorname{Min}\langle v-p, v-p\rangle \text {, s.t. } p \in \mathbb{A}, p(N)=v(N) .
$$

This is of course the same as minimizing the norm $\|v-p\|=\sqrt{\langle v-p, v-p\rangle}$ with the same restrictions.

Theorem 3 For each $v \in \mathbb{V}$, the solution $f(v)$ to the problem (8) exists uniquely. The function $f: \mathbb{V} \longrightarrow \mathbb{A}$ is efficient, linear and has the inessential game property. 
Proof. Choose $v \in \mathbb{V}$ arbitrarily. Let $M(v)=\{p \in \mathbb{A} \mid p(N)=v(N)\}$, and note that $M(v)$ is a linear manifold in $\mathbb{A}$. By the Classical Projection Theorem (Luenberger 1969, p.51), the programme (8) has a unique solution $f(v)$, and $f(v)$ is the only $p \in M(v)$ having the property

$$
\langle v-p, q-p\rangle=0, \text { for all } q \in M(v)
$$

The solution $f$ is efficient by definition. If $p \in \mathbb{A}$ and $w=v+p$, then clearly $f(w)=f(v)+p$, so $f$ has the inessential game property.

Let $w=v+v^{\prime}$ for some $v^{\prime} \in \mathbb{V}$. We want to show that $f(w)=f(v)+f\left(v^{\prime}\right)$, to establish additivity of $f$. So it suffices to show that equation (9) holds for all $q \in M(w)$, when $v$ in that equation is replaced by $v+v^{\prime}$ and $p$ is replaced by $f(v)+f\left(v^{\prime}\right)$. Take an arbitrary $p \in M(v)$, and note that $q-p \in M\left(v^{\prime}\right)$, when $q \in M(w)$. Similarly, $q-p^{\prime} \in M(v)$ when $q \in M(w), p^{\prime} \in M\left(v^{\prime}\right)$. With these substitutions, the left hand side of equation (9) gets the following form:

$\left\langle v+v^{\prime}-f(v)-f\left(v^{\prime}\right), q-f(v)-f\left(v^{\prime}\right)\right\rangle=\left\langle v-f(v), q-f(v)-f\left(v^{\prime}\right)\right\rangle+$ $\left\langle v^{\prime}-f\left(v^{\prime}\right), q-f(v)-f\left(v^{\prime}\right)\right\rangle$.

The first term of the sum on the right hand side of this equation is zero, because $q-f\left(v^{\prime}\right) \in M(v)$ and $f(v)$ solves (8) for $v$. The second term of the sum is also zero, because $q-f(v) \in M\left(v^{\prime}\right)$ and $f\left(v^{\prime}\right)$ solves (8) for $v^{\prime}$. Therefore $f$ is additive.

If $w=a v$ for some $a \in \mathbb{R}$, then obviously $f(w)=a f(v)$, and therefore $f$ is linear. Q.E.D.

Note that if we solve the problem (8) without demanding efficiency $p(N)=$ $v(N)$, the solution would be the orthogonal projection $p(v)$ of $v$ into $\mathbb{A}$ with respect to the inner product $\langle\cdot, \cdot\rangle$. The solution $f(v)$ of $(8)$ is then obtained by taking the orthogonal projection of $p(v)$ w.r.t. $\langle\cdot, \cdot\rangle$ into $M(v)=\{p \in \mathbb{A} \mid$ $p(N)=v(N)\}$.

Our next result states that to any linear solution $f$ that has the inessential game property, there is an inner product $\langle\cdot, \cdot\rangle$ such that $f(v)$ solves (8) for all games $v$.

Theorem 4 Let $f: \mathbb{V} \longrightarrow \mathbb{A}$ be any linear solution that has the inessential game property. Then there is an inner product $\langle\cdot, \cdot\rangle$ such that $f(v)$ solves (8) for all games $v \in \mathbb{V}$. 
Proof. Recall that $\mathbb{A}^{\perp}$ is the orthogonal complement of $\mathbb{A}$ w.r.t. the dot product, and that $v$ can be expressed as a sum $v=v^{\perp}+p^{v}$ in a unique way, $v^{\perp} \in \mathbb{A}^{\perp}, p^{v} \in \mathbb{A}$. The projection map $v \longrightarrow v^{\perp}$ is linear, so there is a matrix $Q$ such that $Q v=v^{\perp}$ for all $v \in \mathbb{V}$. The matrix $Q$ is symmetric and idempotent: $Q^{T}=Q, Q Q=Q$, for all games $v$ (see Luenberger 1969).

Since $f$ is a linear solution, it has a matrix $F: F v=f(v)$, for all games $v$. Define

$$
\langle v, w\rangle=v^{T}\left[F^{T} F+Q\right] w, \text { for all games } v, w \in \mathbb{V} .
$$

This is an inner product, since the matrix $F^{T} F+Q$ is symmetric and positive definite. Symmetry is clear, so let us check positive definiteness. Let $v$ be any game such that $v(S) \neq 0$ for at least one coalition $S$. Then $v^{T}\left[F^{T} F+Q\right] v=$ $f(v)^{T} f(v)+\left(v^{\perp}\right)^{T} v^{\perp} \geq 0$. The value is exactly zero only if $f(v)=\overline{0}$ and $v^{\perp}=\overline{0}$. If $v^{\perp}=\overline{0}$, then $v \in \mathbb{A}$, but in this case $f(v)=v$ since $f$ has the inessential game property, so $f(v) \neq \overline{0}$. Therefore $v^{T}\left[F^{T} F+Q\right] v>0$, and $\langle\cdot, \cdot\rangle$ is an inner product on $\mathbb{V}$.

Take any game $v$. Then

$$
\langle v-f(v), v-f(v)\rangle=f(v-f(v))^{T} f(v-f(v))+\left(v^{\perp}\right)^{T} v^{\perp}=\left(v^{\perp}\right)^{T} v^{\perp}
$$

since $f$ is linear and has the inessential game property. If $p \neq f(v)$ is an arbitrary additive game, then

$$
\langle v-p, v-p\rangle=(f(v)-p)^{T}(f(v)-p)+\left(v^{\perp}\right)^{T} v^{\perp} .
$$

Hence $\langle v-f(v), v-f(v)\rangle\langle\langle v-p, v-p\rangle$, if $p \neq f(v)$, and $f(v)$ solves the problem (8). Q.E.D.

\section{Least square values and the core}

The following example shows that the least square value $L$ is not always in the core of a convex game.

EXAMPLE 1. Let $N=\{1,2,3,4\}$, and define $v$ in the following way. For single person coalitions, $v_{i}=0$ for $i<4$, and $v_{4}=1$. If $S \subset\{1,2,3\}$ and $|S|>1$, then $v(S)=-5+4|S|$. If $4 \in S$ and $S \neq N$, then $v(S)=$ $v(S \backslash\{4\})+1$. The value of the grand coalition is $v(N)=v(\{1,2,3\})+1+1 / 4$. 
The marginal contribution $v(S)-v(S \backslash\{i\})$ is nondecreasing in $S$ for each player $i$, so $v$ is convex. The Banzhaf values are $\beta(v)_{i}=(20+1 / 4) / 8$ for $i<4$, and $\beta_{4}=(8+1 / 4) / 8$. The least square values for players are calculated by $L(v)_{i}=\beta(v)_{i}+[v(N)-\beta(v)(N)] / 4$. These values are $L(v)_{i}=39 / 16$ for $i<4$, and $L(v)_{4}=15 / 16$. Since $L(v)_{4}=15 / 16<1=v_{4}, L(v)$ is not in the core of $v$.

Let $C(v)$ be the core of the game $v$. The core satisfies the additivity condition $C(v+w)=C(v)+C(w)$, if both $C(v)$ and $C(w)$ are nonempty. If $C(v) \neq \emptyset$, then $C(v+p)=C(v)+\{p\}$ for any additive game $p$, since $C(p)=$ $\{p\}$ for additive games. Recall that $v=v^{\perp}+p^{v}$, and that $L(v)=L\left(v^{\perp}\right)+p^{v}$. So if $L(v) \notin C(v)$, this happens because $L\left(v^{\perp}\right) \notin C\left(v^{\perp}\right)$. Since $L$ divides the sum $v^{\perp}(N)$ evenly among the players, $L\left(v^{\perp}\right)(S)=s v^{\perp}(N) / n<v^{\perp}(S)$ for some $S$, iff $L(v) \notin C(v)$.

One could modify the least square value $L$ so that it picks an element from the core whenever the core is nonempty, and if the core is empty then the solution is given by the usual least square value. A natural candidate for this kind a modified least square value is such that it picks the core element that is closest to the game with respect to the Euclidean norm. That is, if $C(v) \neq \emptyset$, then the minimization problem is the following

$$
\operatorname{Min} \sum_{S \subset N}(v(S)-x(S))^{2}, \text { s.t. } x \in C(v)
$$

Since $C(v)$ is convex and compact, a unique solution to this problem always exists. Define the modified least square value $\hat{L}$ by $\hat{L}(v)$ solves $(11)$ if $C(v) \neq$ $\emptyset$, and $\hat{L}(v)=L(v)$ otherwise.

By the Pythagorean Theorem, the solution to (11) can be found by first solving $L(v)$, and then finding the point $x \in C(v)$ that is closest to $L(v)$. Then $x=\hat{L}(v)$. Since $L(v)=L\left(v^{\perp}\right)+p^{v}$, the solution can also be found by finding first $y \in C\left(v^{\perp}\right)$ that is closest to $L\left(v^{\perp}\right)$, and then $y+p^{v}=\hat{L}(v)$. Recall that $L\left(v^{\perp}\right)=v^{\perp}(N) u$, where $u$ is the uniform additive game. Hence $y=\hat{L}\left(v^{\perp}\right)$ is the point in $C\left(v^{\perp}\right)$ that is closest to the equal division of the worth $v^{\perp}(N)$.

One can show that $\hat{L}\left(v^{\perp}\right)$ is actually a Lorenz undominated member of the core of $v^{\perp}$ in the sense of Dutta and Ray (1989). Moreover, if $v$ is convex then $v^{\perp}$ is convex, and $\hat{L}\left(v^{\perp}\right)$ Lorenz dominates every other member of $C\left(v^{\perp}\right)$. But the Dutta-Ray solution (the set of Lorenz undominated members of the 
core) is not additive. Namely, the Dutta-Ray solution of a convex game $v$ need not be the same as $\hat{L}\left(v^{\perp}\right)+p^{v}$, although $\hat{L}\left(v^{\perp}\right)$ is the Dutta-Ray solution of $v^{\perp}$ and $p^{v}$ obviously is the Dutta-Ray solution of $p^{v}$. The modified least square value $\hat{L}$ has the property $\hat{L}(v+p)=\hat{L}(v)+p$, if $p \in \mathbb{A}$, although it doesn't satisfy additivity either. 


\section{REFERENCES}

Dubey, P., Neyman, A., and Weber, J. (1981) Value Theory without Efficiency. Mathematical Operations Research 4, 99-131.

Dutta, B., and Ray, D. (1989) A Concept of Egalitarianism under Participation Constraints. Econometrica 57, 615-635.

Keane, M. (1969) Some Topics in $N$-Person Game Theory. Ph.D. dissertation, Northwestern University, Evanston, IL.

Luenberger, D. (1969) Optimization by Vector Space Methods. Wiley, New York.

Ruiz, L.M., Valenciano, F., and Zarzuelo, F.M. (1996) The Least Square Prenucleolus and the Least Square Nucleolus. Two Values for TU Games Based on the Excess Vector. International Journal of game Theory 25, 113134.

Ruiz, L.M., Valenciano, F., and Zarzuelo, F.M. (1998) The Family of Least Square Values for Transferable Utility Games. Games and Economic Behavior 24, 109-130.

Shapley, L.S. (1953) A Value for N -Person Games. Annals of Mathematic Studies 28, 307-317.

Winter, E. (2002) The Shapley Value. Chapter 53 inn Handbook of Game Theory, Vol. 3, Aumann, R. and Hart, S. (eds), Elsevier, Amsterdam 